\title{
Arbeitsgemeinschaften der DGU (AG Ambulante Urologie)
}

Urologe 2020 · 59:1280-1282

https://doi.org/10.1007/s00120-020-01342-2

() Springer Medizin Verlag GmbH, ein Teil von Springer Nature 2020

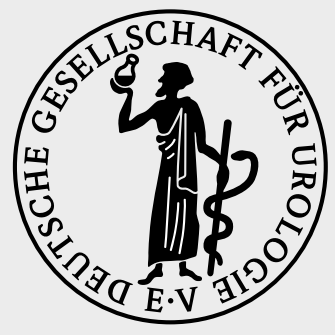

Herausgegeben vom Vorstand der

Deutschen Gesellschaft für Urologie

Schriftführer

Prof. Dr. med. C. Wülfing, Hamburg

Schriftleitung

Rechtsanwalt F. Petersilie, LL.M. (V.i.S. d.P.)

Geschäftsstelle der Deutschen Gesellschaft für Urologie e.V.

Uerdinger Str. 64

40474 Düsseldorf
Joerg Neymeyer ${ }^{1} \cdot$ Thomas Bartelheimer $^{2} \cdot$ Zenai Almedom $^{2}$. Sarah Weinberger ${ }^{1}$. Diana-Elena Moldovan ${ }^{1}$ - Frank König ${ }^{3}$ • Ulf Krapfenbauer $^{4} \cdot$ Thorsten Schlomm $^{1} \cdot$ Karen Weisshaupt $^{5}$ ${ }^{1}$ Charite - Universitätsmedizin Berlin, Klinik für Urologie, Berlin, Deutschland

2Praxis - Urologie am Kurfürstendamm, Berlin, Deutschland ${ }^{3}$ ATURO - Praxis für Urologie, Berlin, Deutschland ${ }^{4}$ Praxis -, urologie am tierpark', Berlin, Deutschland ${ }^{5}$ Charite - Universitätsmedizin Berlin, Klinik für Geburtsmedizin, Berlin, Deutschland

\section{Ergebnisse urologischer Versorgungsforschung}

\section{Nutzen ultramobiler Wireless-Ultraschallsonden bei der täglichen ambulanten und stationären Arbeit}

\section{Einführung}

Entwicklungen immer kleinerer, leichterer und leistungsfähigerer ultramobiler Ultraschallsonden erweiterten die klinischen Einsatzgebiete. Portable Ultraschallgeräte waren 2011 noch ein VScan der 1. Generation mit einem Schallkopf, 2012 war es das portable Ultraschallgerät von Samsung (12 kg) und im Jahr 2020 sind es drei drahtlose koppelbare ultramobile Wireless-Ultraschallsonden, d. h. Wireless Ultrasound Probes (WUPs). Die aktuellen WUPs erweiterten die diagnostische Breite durch die Anwendung abdominaler, linearer und endoluminaler Sonden.

Portable mobile Ultraschallgeräte erwiesen sich in der klinischen Anwendung als praktikabel. Deren Nutzen wurde in der Notfallversorgung und im klinischen Alltag vielfach bewiesen. Besonders unter Pandemiebedingungen erwiesen sich die WUPs in den Mobile Urologists Bag (MUB) als sehr hilfreich. Schrittweise wurden die Gebrauchswerte verbessert und das Gesamtgewicht des MUB reduziert. Die aktuelle „MUB - III. Generation“ (2020) hat nunmehr nur noch ein Gesamtgewicht von 5,2 kg (• Abb. 1).

\section{Zielstellung}

Innerhalb einer Urologischen Versorgungsstudie unter Pandemiebedingungen wurde bereits eine erste Evaluation durchgeführt. Gefragt wurde nach Erfahrungen und Nutzen beim ambulanten Einsatz der

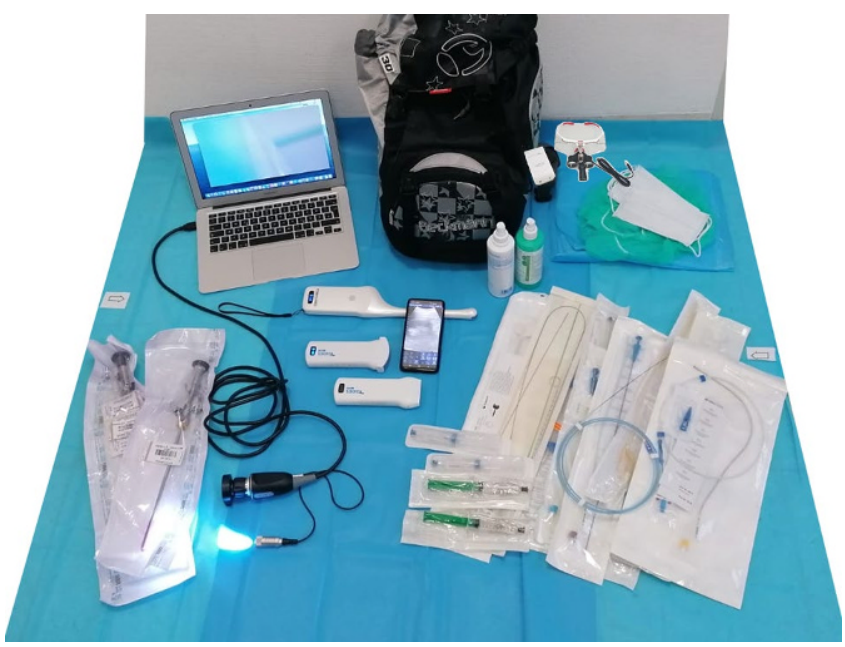

Abb. 1 A Mobile Urologists Bag (MUB) der III. Generation 2020 mit urologischen Basisgeräten und Stents für die ambulante und stationäre Notfallversorgung

mobilen WUPs bei ambulanten Versorgungen potentiell infektiöser Patienten, also unter aktuellen CoVid-19-Hygienekriterien.

\section{Material und Methoden}

Die batteriebetriebenen WUPs, Mides C3cs, L7cs und V7, wurden im klinischen Einsatz bei Visiten, im Operationssaal, in der Notaufnahme und bei Hausbesuchen zur mobilen sonographischen Diagnostik verwendet. Aber auch bei Punktionen, Nephrostomie- oder Zystofixanlagen kamen WUPs zum Einsatz. Evaluiert wurden eine abdominale, lineare und endoluminare Ultraschallsonde. Zwischen 10/2018-08/2020 erfolgten mittels WUP im ambulanten und stationären Bereich insgesamt 792 Untersuchungen.

Die Sonden wurden mittels einer freiverfügbaren SonographieApp (WirelessUSG) über ein gekoppeltes IOS- oder Android-Smartphone oder Tablet bzw. über einen PC bedient. Die Bilddokumentation erfolgte im DICOM, JPEG oder MP4-Format.

\section{Ergebnisse}

Als besonders vorteilhaft erwies sich im klinischen Alltag die schnelle Start- und Betriebsbereitschaft der Sonden (Boot- und Kopplungszeit ca. $20 \mathrm{sec}$.) sowie die lange Akkulaufzeit von 2-3 h im Dauerbetrieb und bei gezieltem Einsatz von 1-2 Tagen. Die Bedienung ist mit Touchscreen des Smartphones oder Tablets einfach. Die Sonographie- 
Tabelle: Kabellose Wifi Sonden 2.4-5 Gigaherz - Ausschließlich Single-User-Zugriff/WPA2

\begin{tabular}{|c|c|c|c|c|c|c|}
\hline Sondentyp & Frequenzbereich & Eindringtiefe & Modus & Dimension & Gewicht & Laufzeit \\
\hline $\begin{array}{l}\text { Abdominal } \\
\text { C3cs }\end{array}$ & $3.5 \mathrm{MHz}$ & $100-200 \mathrm{~mm}$ & $\begin{array}{l}\text { B, B/M, color/ } \\
\text { PW/PDI }\end{array}$ & $155 \times 65 \times 20 \mathrm{~mm}$ & $308 \mathrm{~g}$ & $3 \mathrm{~h}$ \\
\hline $\begin{array}{l}\text { Linear } \\
\text { L7cs }\end{array}$ & 7.5-10 MHz & $20-55 \mathrm{~mm}$ & $\begin{array}{l}\text { B, B/M, color/ } \\
\text { PW/PDI }\end{array}$ & $155 \times 65 \times 20 \mathrm{~mm}$ & $200 \mathrm{~g}$ & $4 \mathrm{~h}$ \\
\hline $\begin{array}{l}\text { Endoluminar } \\
\text { V7 }\end{array}$ & $6.5 \mathrm{MHz} / 8 \mathrm{MHz}$ & $40,60,80,100 \mathrm{~mm}$ & B, B/M, PW/PDI & $270 \times 60 \times 20 \mathrm{~mm}$ & $250 \mathrm{~g}$ & $3 \mathrm{~h}$ \\
\hline
\end{tabular}

App (WirelessUSG) für das iPhone/iPad lief geringfügig stabiler als bei Android-Geräten.

Vergleichbar zu stationären Ultraschallgeräten sind die Bildwiederholsequenzen und Auflösungen der mobilen Sonden zwar geringer, sie sind für den ambulanten oder notfallmäßigen Einsatz aber angemessen. Mehrere Autoren wiesen bereits in Publikationen auf die Qualität der Bildgebung und Genauigkeit der Messungen wie Restharn, Harnstauung und Volumenbestimmung der mobilen Sonden hin. Diese Qualitäten konnten wir bestätigen. Vergleichende Volumenvergleichsbestimmungen der Prostata konnten bei $n=25$ Patienten jeweils am Hitachi Arietta V70 und der Endoluminalsonde Mides V7 zeigten Abweichung der Volumenbestimmung im Intervall von $\pm 4.5 \mathrm{ml}$ und Messungen der Restharnmengen $n=30$ lagen im Intervall von $\pm 25 \mathrm{ml}$. Auch andere Autoren konnten diese Abweichungen ebenfalls beobachten.

Eine Beurteilung der Niere und deren Stauungsgrade wurden mittels portabler Sonden ebenso sicher wie mit Standardgeräten erkannt. Darüber hinaus konnte eine sichere Diagnostik des Urogenitaltraktes und inneren Genitale der Frau erfolgen. Eine spezifisch urologische Diagnostik des Urogenitaltraktes wie bisher mit Standard-Ultraschallgeräten oder portablen High-End-Geräten ist mit den ultramobilen drahtlos koppelbaren Ultraschallsonden (WUP) (siehe Tabelle: Kabellose Wifi Sonden ...) ebenfalls möglich. Beispielsweise kann eine zuverlässige Harnleiterdiagnostik - hier in „Kung-Fu-Technik“ - zum
Nachweis eines prävesikalen Harnleiterkonkrementes ( $\bullet$ Abb. 2) oder eines abgesetzten Harnleiters 3,5 cm oberhalb des Ostiums nach Hysterektomie mit Urinombildung und anschliessender operativer Versorgung mittels „Utereric Bridging“ mittels vollummantelndem Allium-Stentes erfolgen ( $\bullet$ Abb. 3). Darstellungen typischer Bilder wie die eines papillären Blasentumors und einer Harnblasenendometriose sind bei gefüllter Blase ebenfalls sichtbar (• Abb. 4).

Besonders unter klinischen und ambulanten Hygienekriterien der CoViD-Pandemie war die Aussagekraft der Bildgebung der mobilen Sonden (WUP) hoch. Wegen der kompakten Gestaltung der kabellosen Sonden, konnten diese zur Diagnostik und Therapie (Punktion) bedarfsweise mit einem (unsterilen oder sterilen) Latex-Handschuh schützend umschlossen werden. Die Bedienung erfolgte ausschließlich über Knöpfe an der Sonde. Steriles Arbeiten und Infektionsvermeidung ist so möglich. Diese Verwendung eines Handschuhs ermöglicht sicheres Arbeiten, reduziert Verbrauchsmaterial und Kosten. Mit einem sterilen Überzug konnten die Sonden auch im operativen Einsatz und bei Punktionen genutzt werden.

Ein erläuterndes Video wurde beim 50. International Continence Society (ICS) in Las Vegas 2020 vorgestellt (ICSCOVID-19 global epidemic- „Mobile Urologist's Bag III“ (MUBIII)- safety modifications; https://www.ics.org/2020/abstract/613). (• Abb. 5)
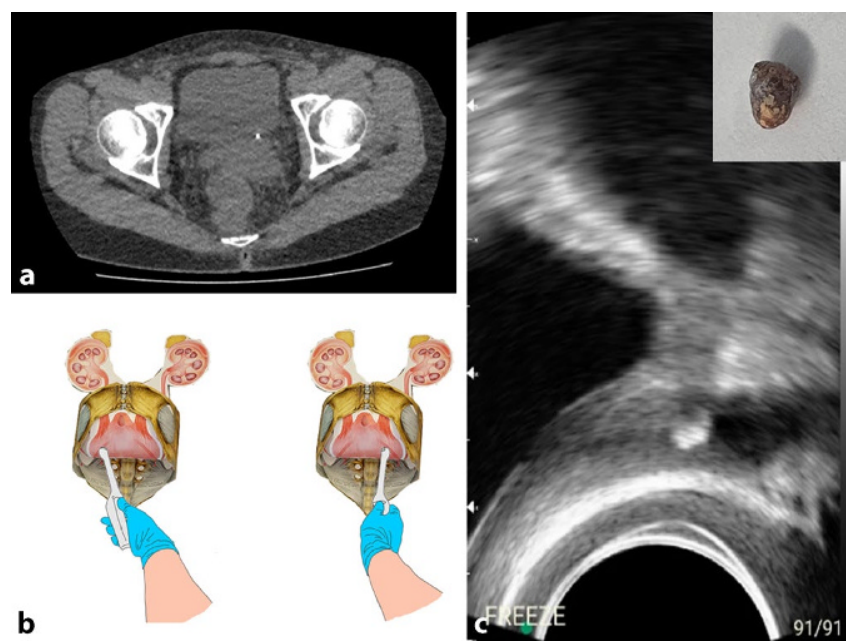

Abb. $2 \Delta$ Diagnostik des prävesikalen Harnleitersteines mittels „Kung-FuTechnik". a CT $3 \mathrm{~mm}$ prävesikaler Stein. b Darstellung der Harnleiterdiagnostik mittels „Kung-Fu-Technik“ - Visualisierung des rechten Harnleiters durch $45^{\circ}$ Drehung des Schallkopfes nach rechts, Visualisierung des linken Harnleiters durch $45^{\circ}$ Drehung des Schallkopfes nach links. c US Bild mit der Endoluminalsonde V7

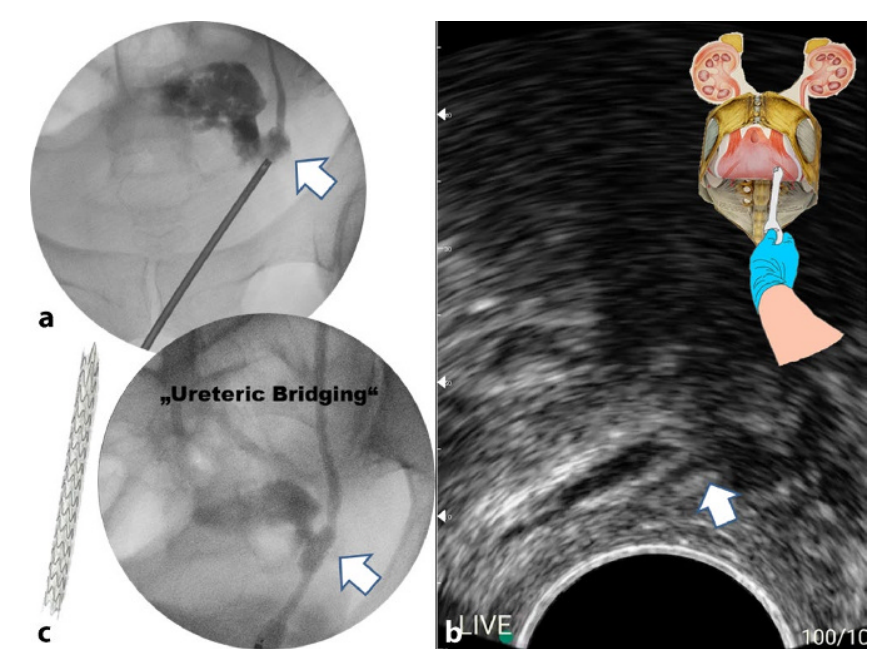

Abb. 3 \& Diagnostik und Therapie distaler Harnleiterlaesionen. a abgesetzter Harnleiter (Thermoschaden) nach abdominaler Hysterektomie. b Darstellung des abgesetzten Harnleiters $4 \mathrm{~cm}$ ab Ostium. c Direkte Versorgung mittels "Ureteric Bridging" mittels vollummantelnden Allium-Stentes eines $2 \mathrm{~cm}$ Defektes 


\section{Arbeitsgemeinschaften der DGU (AG Ambulante Urologie)}

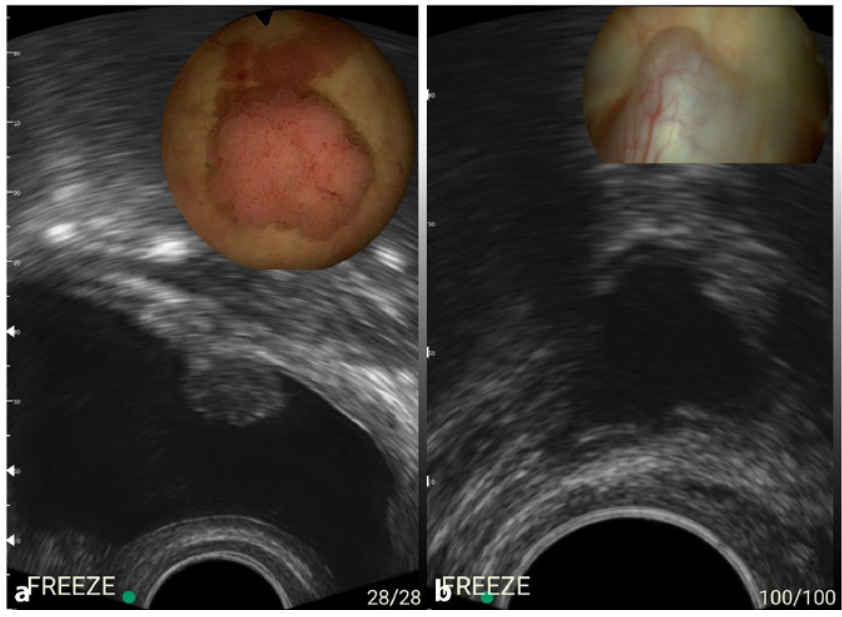

Abb. $4 \Delta$ a papillärer Harnblasentumor an der Hinterwand. b infiltrierende Blasenendometriose oberhalb des Trigonums
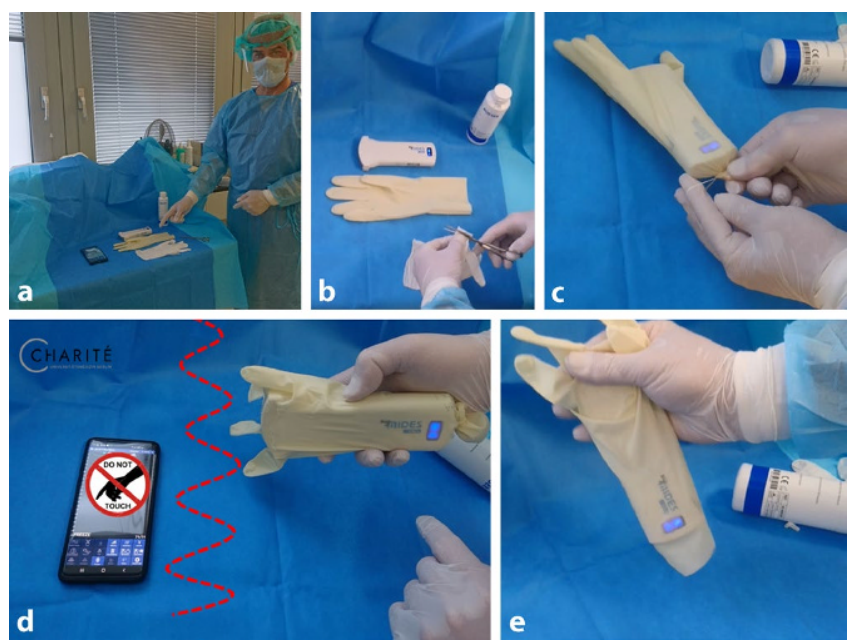

Abb. 5 ム Empfohlene Vorgehensweise zur Ultraschalluntersuchung bei kontagiösen Patienten. a Benötigt werden eine Ultraschallsonde, 1 Paar Handschuhe (im Bedarfsfall im OP steril), Handy/Tablet. b Mittels Schere wird aus einem Handschuh (Finger) ein Verschlussgummi geschnitten. c Der Handschuh wird mit Kontaktgel gefüllt, die Sonde eingeführt und mittels Verschlussgummi geschlossen. $\mathbf{d}$ Die Sonde wird nur über die Knöpfe zum Speichern der Bilder genutzt. e Öffnen des Verschlussgummi und die Sonde kann kontaminationsfrei herausgleiten

\section{Diskussion}

Mittels der WUP verbesserten sich klinische und besonders ambulante Arbeitsabläufe und ermöglichten so schnellere Therapieentscheidungen. Die mittlere Zeitersparnis betrug in der Klinik und Praxis pro Tag ca. $1.5 \mathrm{~h} / \mathrm{d}$ bei der „Morgenvisite“ durch „Bedside-Ultrasound“ und/oder direkte Vorort-Intervention bei Hausbesuchen oder angeforderten konsiliarischen Untersuchungen.
Außerhalb der urologischen klinischen Infrastruktur benötigt ein Facharzt eine urologische Mindestausstattung hauptsächlich für

- Konsiliarische Untersuchungen in fachfremden und urologisch nicht ausgestatteten klinischen Bereichen,

- zur urologische Notfallversorgung „vor Ort“ oder beim Hausbesuch und

- zur urologischen Notfallaufnahme in der regulären Notaufnahmestation.

Besonders unter den hohen Hygieneanforderungen während der CoViD-Pandemie zeigten die neuen WUPs ihren hohen diagnostischen Wert und ihre verbesserten Gebrauchswerte durch zeitoptimiertes dezentrales Arbeiten mit geringstem Kontakt (geschützte Sonden). Dies schließt die sichere urologische Erst- und Nachbetreuung in Senioren- und Pflegeeinrichtungen mit ein.

\section{Fazit}

Ultramobile Wireless-Ultraschallsonden (WUP) als Basisgeräte der Mobile Urologists Bag sichern die unmittelbare Aktionsfähigkeit der Sonographie und so eine rasche Erstbehandlung. Beim sterilen Arbeiten erwies sich das einfache, kostengünstige Arbeiten mittels durch einen die kompakte Ultraschallsonde schützenden Handschuh als vorteilhaft.

Die Ergebnisse der ersten Versorgungsdaten waren für die KV Berlin gut genug, um bereits der ersten Praxis in Berlin bereits eine Abrechnungsgenehmigung für die WUP-generierten Ultraschallleistungen zu erteilen.

Literatur beim Erstautor Dr. J. Neymeyer

\section{Korrespondenzadresse}

\section{Dr. J. Neymeyer}

Sektionsleiter Urogynäkologie, Charité, Universitätsmedizin Berlin Hindenburgdamm 30, 12203 Berlin, Deutschland

joerg.neymeyer@charite.de 